\title{
Multi-Leader Election Algorithm Based On VORONOI Partition for Self-Stabilization in MANETs
}

\author{
T Madhu, S S V N Sarma, J V R Murthy
}

\begin{abstract}
Electing a leader in Mobile Adhoc Networks (MANETs) is the basic problem in providing the stabilization. Many current research outcomes study multi-leader election concept in the static networks and single leader in dynamic networks. The article mainly focusses on multi leader election in dynamic sensor networks where the nodes were installed randomly. This proposed concept elect an exclusive leader when the topology changes occurred eventually over the network. Furthermore, a basic representation used for the process. Also provided accurate proof for the proposed algorithm and shown the way of satisfying the stability condition through ensured results.
\end{abstract}

Keywords: WMSN, Leader Election, Voronoi, Lifetime of Network, Energy Consumption

\section{INTRODUCTION}

A group of mobility nodes form Wireless Mobile adhoc Sensor Networks (WMSN) with robust network without infrastructure. These networks are formed with lesser and low-cost sensors, which determine the location information, later gather and broadcast the message. All the nodes are portable nodes. Self-stabilization is conjectural framework of transmission fault-tolerant. This structure stands many measurable and categorical errors like data loss, memory corruption, and basic network topology abnormalities.

Wireless Mobile adhoc Sensor Networks (WMSN) is distributed and pertain to identify and resist the specified location with an arbitrary or specific method. Wireless Mobile adhoc Sensor Networks (WMSN) are used in various fields like defense purpose weather watching and disaster prevention [1-5][31].

A Wireless Mobile Adhoc Sensor Network (WMSN) isagroup of sensor nodes which carries precise activities. Contrasting the conventional connection oriented, the sensors transfermessagesthrough each and every node handle to reach destination [31].

Election of a leader is one of most important issue for distributed computing, beneficial as a subroutine for applications which needs finding an exclusive processor within many number of contending processors.

The leader election approach requires the range of a leader which starts with primary backup based on replication using fault tolerance in group communication

Revised Manuscript Received on August 14, 2019.

T Madhu, Research Scholar, JNTUK, Kakinada \&Associate Professor, Department of Computer Science and Engineering, SRTIST, Nalgonda, Telangana, India. (E-mail: madhuthallapalli@yahoo.com)

S S V N Sarma, Professor of CSE, Vagdevi College Of Engineering, Warangal, Telangana, India.(E-mail: ssvn.sarma@gmail.com)

J V R Murthy, Professor of CSE JNTUCE, JNTUK, Kakinada, A.P India.(E-mail: mjonnalagedda@yahoo.com) systems [6] and in multiplayer games for video conferencing [7].

Electing a leader is a basic issue in distributed computing for infringementbalance and reviewingadditionalissues, like spreading and aggregation of the data. Only one processor is finalized as the leader in leader election process [8].

The nodes of network are continuously moving from one place to another place for various purposes. Due to this the unpredictable assortment from the varying location of the hops in mobile networks letdown and overhaul of every connections in wireless networks. Most of the recent investigations are majorly concentrated on variousproposals specified above to vigorous networks, which together with sensor and mobile networks. For example, the data delivery fault-prones were found in networks by using replicationbased scheme [9]. This is the one of the reason of the necessity of leader election algorithm in networks in dynamic nature.

Every network maintains a unique leader called as local leader when the topology cease problem occurs while the communication occurring between nodes [10]. The proposed leader election algorithm is the leeway method of the existing algorithm [11], where it is an addition of the routing algorithm in MANETs like TORA[12][9].

Two algorithms for routing wereproposed grounded on the basic idea of reversal link [9].

The final achievement of each and every routing algorithm is to establish convenient routing paths over the network structure from source to destination node in graph concept. A complete ordered set is tomaintain the height variable of every node in those algorithms. Also the communication link is in bidirectional between nodes which are formed from descending order from superior height to lesser height. The source node means the links will be established, and the sink means no outgoing links.

The height is a pair containing of a hostage and exclusive node ID, although in another algorithm the height is threeway having couple of counter values and the id of node[9]. In two algorithms, lexicographically compared with heights throughminimum sub sequential constituent presence of the node id.

The intention of the reference level is to specify the mentioned path to sink if the node lost its path. All the reference level values are zeros at starting position. If the 


\section{MULTI-LEADER ELECTION ALGORITHM BASED ON VORONOI PARTITION FOR SELF-STABILIZATION IN MANETS}

node drops previous departing connection because of the connection moving down the node begins a new reference level by varying the initial component of triplet to existing time, then id of its node is secondly, and zero value at third,revealing the process to find the destination node. Reference levels are spread across a connected thing, as every node drop the out bounding connections because of changes in height, leading to finding the stand by routes towards the sink.

The main thought of TORA wastoimprove leader election [13], if the node finds that partitions of previous leader i.e. sink, after that converting dormant and elects new leader itself. About new leader information spreading over the network with connected component.

The nature of self-stabilization algorithms provides for dynamic nature environment. The algorithm will start stabilization once the change was happened.

In this paper the introduction isprovided in Section -I, the various scenarios and outcomes by parallel researchers with the advantages and disadvantages of the same in Section II, Basic of useful information in Section-III, the evaluation of results was explained in Section IV

\section{RELATED WORK}

The emblematic fields like wireless networks and distributed computing application on the concept of randomization, where the randomized algorithms are naiver and effective over deterministic algorithms. The fundamental approach in distributed network is to electing a leader and many researchers are working on it from past two decades in areas of networks and communication systems. Now it is crucial algorithm for dynamic networks to find the better research outcomes.

Many available proposals areconfined and applied in only deterministic algorithms $[10,11]$. But in practicality, thereis a great difficulty in the execution. The regional consecutive leader election (RCLE) is the extension of conventional MANETs and provided the solution of RCLE in two and three dimensional spaces [10].

In this proposal the node count over the network besides the startup time are identical. Also the algorithm proved the complexity of message bit id $((n(\log n+\log r))$ per node in round $r, n$ is the number of nodes.

After that the amended RCLE outcome proposed[10][11], which needs the nodes with mobility feature should elect a leader within the time as mentioned in the constrained way, and complexity of time of the proposed one is as $\mathrm{O}(\mathrm{Dn})$ where the network diameter represent as D and total number of nodes as $\mathrm{n}$. The another matching algorithm which assures algorithm will terminate in $\mathrm{O}(\mathrm{Dn})$ rounds and the message will send one at a time while broadcasting message per round.

A leader election algorithm[12], for each topology of networks should beconfined to only one leader and this one isbased on TORA. Also correctness of algorithm is validated.

Another protocol of leader election in variable and asynchronous topology which elected leader successfully oncethe topology variations occurred.Additionally many schemes were proposed for leader election algorithms and applying them to mobility environment [13-18].
Various approaches and mechanisms were proposed for dynamic nature networks and theywere also made in very simplified manner [19]. Nevertheless, most of them have similar distinctive conventions like node continuous mobility, and total nodes count and unlimited frequency in mobility nature.

One of the natural characteristic like entering and exiting of the nodes from or to the network topology leads to variations of the network [20]. All of these kind of activities will be occurring in the peer based network. For these the authors have given random distributed leader election algorithm with time in logarithmic multiples, confirming practically ubiquitously to be even underneath in maximum possibility of intervention.

Apart from the leader election issue, many proposals were proposed with the inferior bound of time and message complexities in the stagnant networks.

One of those proposals are inferior bound of time and message complexities likely $\mathrm{O}(\mathrm{n} \log \mathrm{n})$ [21]. The proposal [22], deliberated for the process of electing a leader in complete networks like synchronous and asynchronous.

Some research proposals are concentrating complexities like time and message randomized inherent leader election in distributed synchronous networks [23]. All these nodes in the network will be effected by topology variations because of its dynamic nature. That means any node can enter and exit the network at any point of time.

A random leader election algorithm proposed [24-30] for distributed synchronous networks. In the process of electing a leader by using $\mathrm{O}(1)$ rounds and the complexity of message is $\mathrm{O}(\sqrt{ } \operatorname{nlog} 32 \mathrm{n})$.

\section{PROPOSED METHODOLOGY}

In this work, initially the voronoi diagram is used to divide the entire network into various number of regions. Later on found the solution for redundant nodes for reducing the consumption of energy. Finally designed an algorithm to find the solution for leader election algorithm.

\section{Initial Assumptions of the Model:}

Due to node mobility the topology variations of network is continuous in the period of time is one of the characteristics of dynamic networks. Initially, the mobile network can be represented with $\mathrm{G}=\mathrm{G} 1, \mathrm{G} 2, \mathrm{G} 3, \ldots \ldots, \mathrm{Gn}$, where $\mathrm{Gn}=(\mathrm{Vn}, \mathrm{En})$ is the topology of the network at instance of the time $\mathrm{n}$, in that $\mathrm{Vn}$ and En represent the groups of nodes and Edges, in turn. The two-dimensional space R of poisson'sdistribution is to keep an eye on each and every node which has a distinctive identifier ID.

\section{Problem Definition}

Firstly, each and every node has equal power transmission $\mathrm{P}$, and all nodes can regulate it within is in accessible range, through this we can guarantee that the communication between nodes with less power. Apart from that, assume the nodes can only enter or exit will be done once normally. But the node moves in all the directions in

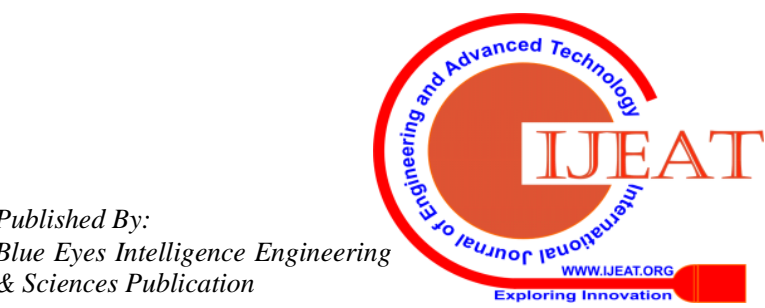


any manner more than once over the network. When the node is represent as heterogeneous means node movement direction is chosen randomly in $[0,2 \pi]$ with the symbol of $v$, the time for maximum movement is represent with $t_{\max }$.

The network divided based on the concept of voronoi diagram and reasons are listed as per:

1. Hexagon network topology applied on regular network nodes because of easy control and flexibility rather than circle, square mesh and other networks.

2. This topology easily works and is effective to contiguous search point by using voronoi diagram.

Additionally the conventional voronoi is located at single area and the leader node along with other nonleader nodes also placed in the same area at that particular time. The partition of the network procedure is mentioned below:

Initially, choose $\mathrm{k}$ nodes in random manner subsequently the initializing the topology of total network and the entire network partitioned as $\mathrm{k}$ areas called it as voronoi-1.

Secondly, a modest leader election algorithm is normally be governed by the ID of nodes in the respective area.

At last repartition of the network is required once the leader election algorithm terminate. Assume various leaders of different $\mathrm{k}$ areas and called them as voronoi- 2 in partitions in the network.

Apart from that the propositions about dynamic networks are:

1. Each and every node have equal sensing power capacity and the range active communication.

2. To create strengthen the attainment of the proposed algorithms for leader election, the knots possess inactive formal indoors approximately rounds.

3. The nodes are symmetric in the communication network.

4. The upper bound of various nodes represents the movement in network represented with ${ }_{\mathrm{Nu}}$ movement.

The parameters are shown in the Table 1 .

\begin{tabular}{|c|c|}
\hline Symbols & Description \\
\hline$n$ & The numter of dead nodes \\
\hline$D$ & The diemanar of network \\
\hline p & The inisal raramistion powa \\
\hline$\lambda$ & The dentiky of lazders \\
\hline$\lambda_{2}$ & The derisy of nonHeadars \\
\hline * & The numter of regiona that paritiared by Vovond \\
\hline$N_{*}$ & The number of neigtibors of node $u$ \\
\hline$k=r$ & The numter of optimel nogions \\
\hline p & 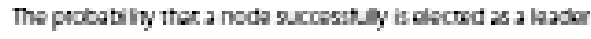 \\
\hline 2 & The sum of the disence bawoan non leasers and leaders \\
\hline N & The numter of nonleaders in the isgon \\
\hline$d$ & The average diatances bawoen nontazders and leadas \\
\hline
\end{tabular}

\section{Node Distribution Model:}

Initially, divide various regions of the network and arrange in the form voronoi cell that the leader is situated in the middle for every region. The Poisson Point Process (PPP) property need to use that means autonomous retreating, for the analysis of consumption of energy of the nodes.

Symbolize the group of leaders and group of non-leaders as Poisson Point Process $\phi_{\mathrm{r}}$ and $\phi_{\mathrm{b}}$ with densities $\lambda_{1}$ and $\lambda_{2}$ in turn.

\section{Consumption of Energy Model and Analysis:}

Basically, sensor contains four components which are for communication, snooze, discovery and evaluation.

This work majorly focusses on controlling the usage of energy to handle the procedure in communication of each and every node in proper manner, so that the overall utilization of energy is very less.

\section{Node Mobility Model:}

The model for mobility is typically mentioned to label the outlines of mobile, like the route, rate, and time variation in node acceleration.

The important role is in finding the performance of algorithmic approach also make the ultimate realistic model to simulate the real world. Previous a number of models for mobility feature are proposed to reinvent the real world settings.Several types of models were proposed for random based mobility. The proposed work, a Random Walk Mobility (RWM) model and Random WayPoint mobility (RWMP) model were considered.

\section{RWM Model:}

This model mobility feature can works without memory management. The result is self-regulating based on the previous positions. The stirring node will move to another location with the route and proportional manner with randomized fashion over the network. The node's rate of movement considered and assumed similar with all other nodes in simulation purpose.

\section{RWPM Model:}

This model is an extension of RWM model with the suspension (Pause) time to alter the direction of movement and rate. The stirring node is hold the motionless at a positon in some duration and this time is called as pause time. The stirring node will choose the sink in specific location after that time. In RWPM model, these time will $b$ finding the feature of mobility of a node. And obviously it effects on the performance of the network.

\section{Algorithm description}

The need of energy efficient algorithms in sensor networks because of the battery life constraint along with the controlled communication overhead and speed of computation. To conquest these issues the Voronoi Leader Election (VLE) algorithm was proposed to fulfil the said requirements of the sensor networks especially in dynamic organized with multiple nodes. The concept of leader election plays a key role, and each and every location of voronoi will be acting as leader after dissolution of the algorithm. All the remaining non-leader nodes will be managed by the leader node in the specific area and also interconnects with rest of the leaders in various areas either in single-hop or multi-hop way.

The leader cannot work properly as mentioned in the below said conditions:

1. If the leader quits the existing cell of voronoi due to its mobility feature, will loose the leader role and act like

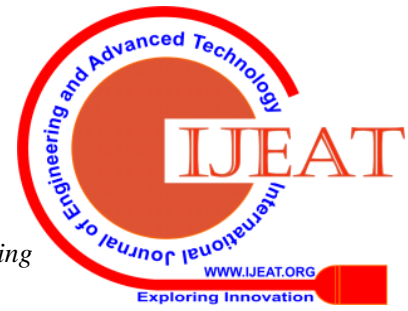


the normal node aka non-leader.

2. If the leader could not have the sufficient threshold energy value the network.

The process needs to implement for new leader election by that time whenever the leader is available for a specific voronoi.

In this proposal the concept of sleeping and walking up was introduced to improve the performance of the network.

Various notations are used in this proposal as mentioned in the below table 2 .

\begin{tabular}{|c|c|}
\hline Symbols & Description \\
\hline Nolath & The number of dead nodes \\
\hline E & The initisl energy af nodes \\
\hline$E_{r e-\infty}$ & The remaining enengy of a lesder \\
\hline Eremo & The remaining energy of a nan-leader \\
\hline it Mowing D & The set of the it ID of moving nodes \\
\hline t & The maving time of a moving node \\
\hline$t_{\min }$ & The maximum time allowing to move \\
\hline$\checkmark$ & The moving rate of a moving node \\
\hline NLe & The nor-leader nodel \\
\hline$S R$ & The sensing radius of nodel \\
\hline$\tau_{\ell}$ & The set of non-lesders with the ascending oeder of it $1 D$ \\
\hline $\mid \pi /$ & The size of $T_{i}$ \\
\hline 8 & The minimurn energy cost when sends ane messsge \\
\hline
\end{tabular}

\section{Algorithm for Election of a Leader:}

A basic algorithm for leader election is depended on voronoi divisions. The various steps of the proposed algorithms is as follows:

\section{Partitioning the Network}

Initially, $\mathrm{n}$ number of nodes are chosen and divided the network area into $\mathrm{n}$ voronoi regions.

\section{Exchange of information}

Each node having Distinctive ID and the same initial energy E. Node x broadcasts ID and distance messages to one hop neighbours. The table of distances of every one-hop node neighbours along with IDs will be formed. After that every node compare with its neighbours IDs.

\section{Node ID Ordering}

Every node keeping order the ID containing with all neighbours along with its own by the descending order.

\section{Leader Election Process}

The node ID $\mathrm{x}$ will complete power to control all other nodes over the network when it is having the maximum neighbours nodes and the residual energy is higher than with minimum of $10 \% \mathrm{E}$. The deathnodes noted when the residual energy $\mathrm{N}_{\text {death }}$. The node cannot obtainable in two cases, i.e. whenever the ID of the the ID of the node lesser than the ID time-honouredfrom neighbours of the ID, and it will be precise by the node has largest ID.

The proposed algorithm procedure steps as mentioned below:

Algorithm Multi-Leader Election Algorithm based on Voronoi Partition

\section{Assumptions:}

The partitions of the network is based on voronoi diagram and travelling all the nodes over the network.

The types of the node state: leader, non-leader.
Finding the dead nodes with the help of the $\mathrm{N}_{\text {death. }}$

\begin{tabular}{|c|c|}
\hline Parameters & Value \\
\hline Selection & $\begin{array}{c}\text { Roulette-Wheel } \\
\text { Selection }\end{array}$ \\
\hline Substitute Paths & $\mathbf{3}$ \\
\hline
\end{tabular}

\section{Level 1: Exchanging the message between nodes}

1. For each node $\mathrm{x}$ the network

2. \{

3. Node $\mathrm{x}$ broadcasts ID and distance messages to one hop neighbours

4. The message obtains from Node $\mathrm{x}$ neighbours.

5. The comparison has been made with all neighbour nodes of Node $\mathrm{x}$.

6. $\}$

7. Every node order the IDs in decreasing way of its all neighbour nodes

Level II:

8. if (Node $x$ ID is bigger than of its neighbors and Ere-le $\geq 10 \% E$ )

9. \{

10. State of the node $\mathrm{x}$ is to be as Leader

11. Node $\mathrm{x}$ is transmit to this message to all its neighbors

12. else if $($ Ere-no $=0)$

13. \{

14. the node dies and Ndeath $=$ Ndeath +1

15. \}

16. else

17. Change the state of $u$ to nonleader

18. \}

\section{EVALUATION OF RESULTS}

The performance of the proposed concept assessed in this section by using the simulation in simulator. The Simulation used for evaluation of the protocols is Network Simulator 3 (NS3). The goal of simulation experiment is to examine influence of multi-leader selection algorithms. The measures employed for assessment the energy values during the node movements Table 3 represents the constraints applied for demonstrating the simulation to analyse the Approaches. Table 4 represents the constraints applied for Leader Election Algorithm.

Table 3: Constraints used for Simulation

\begin{tabular}{|c|c|}
\hline Parameters & Value \\
\hline Simulation Period & $1000 \mathrm{~s}$ \\
\hline \# Experimental Trails & 6 \\
\hline Network Coverage Area & $300 X 300 \mathrm{~m} 2$ \\
\hline Number of Nodes & 100 \\
\hline Routing Zone Radius & 1 \\
\hline Broadcast Range & $100 \mathrm{~m}$ \\
\hline
\end{tabular}


Table 4: Constraints used in multi-leader selection algorithms

\begin{tabular}{|l|l|}
\hline Parameters & Value \\
\hline Selection & $\begin{array}{l}\text { Roulette-Wheel } \\
\text { Selection }\end{array}$ \\
\hline Substitute Paths & 3 \\
\hline
\end{tabular}

Initially, the network with nodes in the region of $300 \mathrm{~m} \mathrm{X}$ $300 \mathrm{~m}$ also few settings described in the table 3 and table 4 . Apart from that the following considerations also to be satisfy to obtain the results:

1. Each and every node must be maintain the mobility rate, battery energy (initially) and movement time to be uniform. The consumption of energy for transfer and receive data to be $0.5 \times 10^{-7}$.

2. The network communication and sensing range should be between 87.7 and $43.8 \mathrm{~m}$.

3. The entire lifetime to be divided into rounds. In the worst case 10 nodes to be participated in moving process in a round. It should be verify for each 10 rounds. The energy depletion for one movement is equal to $0.1 \times 10^{-5}$.

For validation the effect of the rounds in executions of proposed algorithm on energy consumption, initially the total nodes are 100 with the 20 seconds for the movement time at most. In this situation the leader getting the data from its hops and consumption of data in two parts like energy for transfer data and receiving data. Any node movement confined to $300 \mathrm{~m}$ with the speed $5 \mathrm{~m} / \mathrm{s}$. with this the energy consumption is very high.

The way of the decreasing the residual energy of nodes proportionality with the number of rounds which demonstrated through the figure 1 . With this the movement nodes effects the remaining energy of the node. That means the maximum energy will be consumed proportional to the many number of nodes movement over the network.

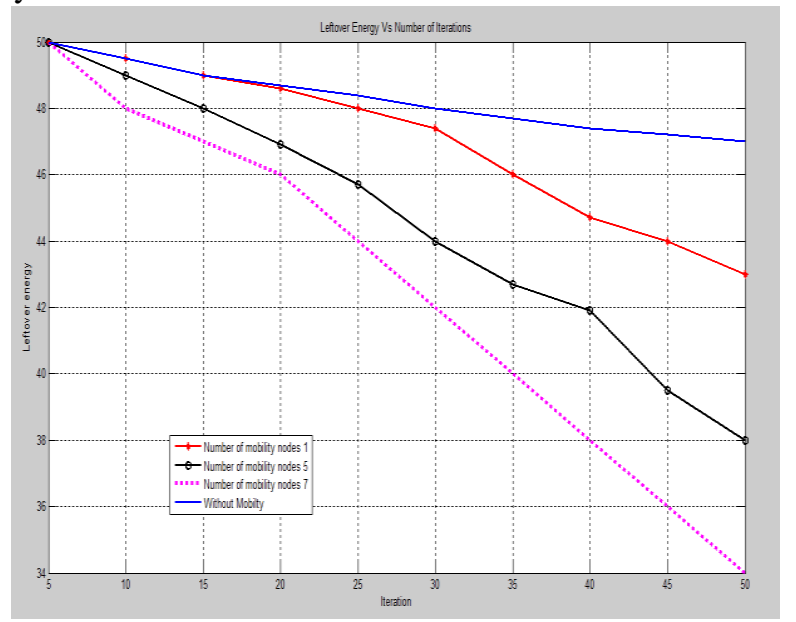

Figure 1. Remaining energy Vs the number of iterations

The figure 2 demonstrating the energy consumption with the impact as per movement time. For every curve shows the energy consumption against the number of moving nodes.

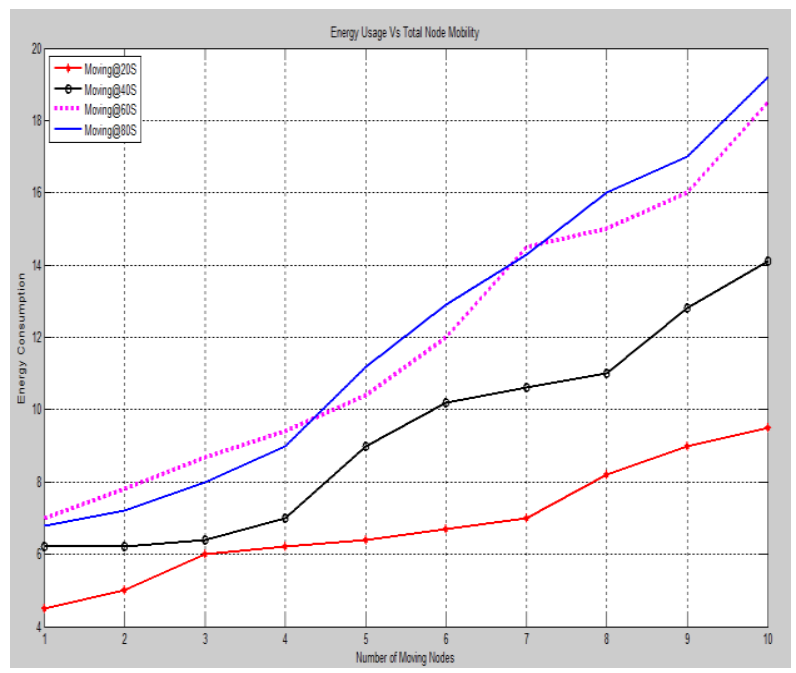

Fig. 2 energy consumption and the number of moving nodes

\section{CONCLUSION}

In the proposed research article, a novel approach is proposed for electing a leader by using the partitioning the entire network based on Voronoi, also make it apply for each cell to run the Multi-Leader Election Algorithm. The issue of fault-tolerant is stated by using the proposed MultiLeader Election Algorithm based on Voronoi Partition. The innovation was made with the help of Voronoi diagram by dividing the various parts of the network, through this model we can the save the energy consumption. Likewise, we improve the network performance by choosing the less number of partitions, so that the optimum resource utilization is achieved and also avoids the energy consumption of the network. For the fault tolerance, we have picked up the current active node whose ID is the largest locally and whose leftover energygratifies the less energy constraint requirement. Need to focus on more efficient concepts to avoid major reduction in energy consumptionin the future.

\section{REFERENCES}

1. J Li, S Cheng, Z Cai, J Yu, C Wang, Y Li, Approximate holistic aggregation in wireless sensor networks. ACM Trans. Sens. Netw. 13(2), 1-24 (2017)

2. A Mainwaring, D Culler, J Polastre, R Szewczyk, J Anderson, in Proc. WSNA'02 workshop. Wireless sensor networks for habitat monitoring, (2002), pp. 88-97

3. J Yu, B Huang, X Cheng, M Atiquzzaman, Shortest link scheduling algorithms in wireless networks under the sinr model. IEEE Trans. Veh.Technol. 66(3), 2643-2657 (2017)

4. Z He, Z Cai, S Cheng, X Wang, Approximate aggregation for tracking quantiles and range countings in wireless sensor networks. Theor. Comput. Sci. 607(3), 381-390 (2015)

5. S Cheng, Z Cai, J Li, H Gao, Extracting kernel dataset from big sensory data in wireless sensor networks. IEEE Trans. Knowl. Data Eng. 29(4), 813-827 (2017)

6. R Rana, C Chou, S Kanhere, N Bulusu, W Hu, in Proc. IEEE IPSN. Ear-phone: an end-to-end participatory urban noise mapping system, (2010), pp. 105-116 
7. D Work, O Tossavainen, Q Jacobson, A Bayen, in Proc. ACC. Lagrangian sensing: traffic estimation with mobile devices, (2009), pp. 1536-1543

8. Z He, Z Cai, J Yu, X Wang, Y Sun, Y Li, Cost-efficient strategies for restraining rumor spreading in mobile social networks. IEEE Trans. Veh.Technol. 66(3), 27892900 (2017)

9. H Attiya, J Welch, Distributed computing: fundamentals, simulations and advance topics. (Wiley, 2004). ISBN:0471453242

10. H Chung, $\mathrm{P}$ Robinson, $\mathrm{J}$ Welch, in Proc. FOMC'10 Workshop. Regional consecutive leader election in mobile ad-hoc networks, (2010), pp. 81-90

11. H Chung, $\mathrm{P}$ Robinson, $\mathrm{J}$ Welch, in Proc. ACM SIGACT/SIGMOBILE FOMC'11 Workshop. Optimal regional consecutive leader election in mobile ad-hoc networks, (2011), pp. 52-61

12. N Malpani, J Welch, N Vaidya, in Proc. of the 4th international workshop on Discrete Algorithms and methods for mobile Computing and Communications. Leader election algorithms for mobile ad hoc networks, (2000), pp. 96-104

13. R Ingram, T Radeva, J Walter, J Welch, in Proc. SPDP. An asynchronous leader election algorithm for dynamic networks, (2009), pp. 1-12

14. K Hatzis, G Pentaris, P Spirakis, V Tampakas, R Tan, in Proc. ACMSPAA. Fundamental control algorithms in mobile networks, (2009), pp. 251-260

15. P Parvathipuram, V Kumar, G Yang, in Proc. ICDCIT. An efficient leader election algorithm for mobile ad hoc networks, (2004), pp. 32-41

16. S Vasudevan, J Kurose, D Towsley, in Proc. IEEE ICNP. Design and analysis of a leader election algorithm for mobile ad hoc networks, (2004), pp. 350-360

17. A Boukerche, $\mathrm{K}$ Abrougui, in Proc. IWCMC. An efficient leader election protocol for mobile networks, (2006), pp. 1129-1134

18. F Kuhn, N Lynch, C Newport, The abstract mac layer. Distrib. Comput. 24(3), 187-206 (2011)

19. F Kuhn, R Oshman, Dynamic networks: models and algorithms. ACM SIGACT News. 42(1), 82-96 (2011)

20. J Augustine, G Pandurangan, $P$ Robinson, $T$ Amitabh, in Proc. ACM-SIAMSDA. Towards robust and efficient computation in dynamic peer-to-peer networks, (2012), pp. 551-569

21. E Korach, S Moran, S Zaks, in Proc. ACM SPDC. Tight lower and upper bounds for some distributed algorithms for a complete network of processors, (1984), pp. 199207

22. Y Afek, E Gafni, in Proc. ACM SPDC. Time and message bounds for election in synchronous and asynchronous complete networks, (1985), pp. 186-195

23. S Kutten, G Pandurangan, D Peleg, A Trehan, in Proc. ACM SPDC. On the complexity of universal leader election, (2013), pp. 100-109

24. S Kutten, G Pandurangan, D Peleg, A Trehan, in Proc. ICDCN. Sublinear bounds for randomized leader election, (2013), pp. 348-362 M Dinitz, J Fineman, S Gilbert, N Calvin, in Proc. ISDC. Smoothed analysis of dynamic networks, (2015), pp. 513-527

25. $\mathrm{J} \mathrm{Yu,} \mathrm{N} \mathrm{Wang,} \mathrm{G} \mathrm{Wang,} \mathrm{Constructing} \mathrm{minimum}$ extended weakly-connected dominating sets for clustering in ad hoc networks. J. Parallel Distrib. Comput. 72(1), 35-47 (2012)

26. J Yu, N Wang, G Wang, D Yu, Connected dominating sets in wireless adhoc and sensor networks-a comprehensive survey. Comput. Commun.36(2), 121134 (2013)

27. J Yu, Q Zhang, D Yu, C Chen, G Wang, Domatic partition in homogeneous wireless sensor networks. J. Netw. Comput. Appl. 37, 186-193 (2014)
28. X Gu, J Yu, D Yu, G Wang, Y Lv, Ecdc: An energy and coverage-aware distributed clustering protocol for wireless sensor networks. Comput.Electr. Eng. 40(2), 384-398 (2014)

29. J Yu, Y Qi, G Wang, Q Guo, X Gu, An energy-aware distributed unequal clustering protocol for wireless sensor networks. Int. J. Distrib. Sensor Netw. 2011, 1-8 (2011)

30. S Foss, S Zuyev, On a Voronoi aggregative process related to a bivariate poisson process. Adv. Appl. Probab Univ. Sheffield. 28(4), 965-981 (1996)

31. M V Narayana, Aparnarajesh Atmakuri“A-ZHLS: adaptive ZHLS routing protocol for heterogeneous mobile adhoc networks" International Journal of Engineering \& Technology, Volume 7 Issue 3(2018), PP.1626- 1630-ISSN: 2227-524X.

\section{AUTHORS PROFILE}

T MADHU, Research Scholar, JNTUK, Kakinada \&Associate Professor, Department of Computer Science and Engineering, SRTIST, Nalgonda.

E-mail: madhuthallapalli@yahoo.com

S S V N SARMA, Professor of CSE, Vagdevi College Of Engineering, Warangal

E-mail:ssvn.sarma@gmail.com

J V R MURTHY, Professor of CSE JNTUCE, JNTUK, Kakinada

E-mail: mjonnalagedda@yahoo.com. 